\title{
Seroma-associated primary anaplastic large- cell lymphoma adjacent to breast implants: an indolent T-cell lymphoproliferative disorder
}

\author{
Anja C Roden ${ }^{1}$, William R Macon ${ }^{1}$, Gary L Keeney ${ }^{1}$, Jeffrey L Myers ${ }^{2}$, Andrew L Feldman ${ }^{1}$ \\ and Ahmet Dogan ${ }^{1}$ \\ ${ }^{1}$ Department of Laboratory Medicine and Pathology, Mayo Clinic, Rochester, MN, USA and ${ }^{2}$ Department \\ of Pathology, University of Michigan Health System, Ann Arbor, MI, USA
}

\begin{abstract}
Non-Hodgkin lymphomas of the breast are rare, encompassing approximately $0.04-0.5 \%$ of all malignant breast tumors, and the vast majority are B-cell lymphomas. In contrast, Iymphomas of T-cell phenotype have been rarely reported and some of these have been in close proximity to a breast implant. In our consultation practice, we have identified four patients with primary T-cell anaplastic large-cell lymphoma presenting adjacent to silicone or saline breast implants. All patients presented with seroma and neoplastic cells were identified in suspension in the serous fluid without solid tissue invasion. Three patients had no evidence of systemic disease (stage 1E), and one patient was not staged. The mean age of the patients was 46 years (range, 34-59 years). In all patients, the neoplastic cells had a T-cell phenotype, expressed CD30, cytotoxic granuleassociated proteins, EMA and clusterin, and were anaplastic lymphoma kinase-1-negative. Clonal T-cell receptor $\gamma$-chain gene rearrangements were identified in three patients. All patients underwent capsulectomy with removal of the implant. One patient subsequently received chemotherapy and radiation therapy, and another was treated with radiation alone. The third patient received no further therapy and the fourth patient has been recently diagnosed. After a mean time of 13 months (range, 9-20 months), all three patients with follow-up were alive and well without any recurrence or systemic disease. Although the follow-up time was relatively short, our series and other reported cases suggest that primary anaplastic large-cell lymphoma adjacent to breast implants is an indolent T-cell lymphoproliferative disorder.

Modern Pathology (2008) 21, 455-463; doi:10.1038/modpathol.3801024; published online 25 January 2008
\end{abstract}

Keywords: anaplastic large-cell lymphoma; primary lymphoma of breast; breast implant; silicone; saline

Non-Hodgkin lymphomas of the breast are rare and comprise approximately $0.04-0.5 \%$ of all malignant breast tumors and $2.2 \%$ of extranodal lymphomas. ${ }^{1,2}$ The vast majority of breast lymphomas are B-cell lymphomas. T-cell lymphomas of the breast have been reported rarely, and few of these occur without an associated implant. ${ }^{3,4}$ In recent years, there have been several case reports of patients developing lymphomas in close proximity to silicone or saline breast implants. ${ }^{5-13}$ Interestingly, the majority of implant-associated lymphomas were T-cell lymphomas, including anaplastic large-cell lymphoma $(n=5)^{5-7,13}$ and mycosis fungoides/Sézary syndrome $(n=4){ }^{8,9}$ In contrast, only three B-cell lymphomas

Correspondence: Dr A Dogan, MD, PhD, Department of Laboratory Medicine and Pathology, Mayo Clinic, Hilton 11, 200 First Street SW, Rochester, MN 55905, USA.

E-mail: Dogan.ahmet@mayo.edu

Received 7 August 2007; revised 4 October 2007; accepted 8 October 2007; published online 25 January 2008 were described, including a primary effusion lymphoma, a follicular lymphoma and a lymphoplasmacytic lymphoma. ${ }^{10-12}$ Furthermore, two of the patients with B-cell lymphomas presented with systemic disease at the time of diagnosis. ${ }^{11,12}$

In this study, we present four patients with primary anaplastic large-cell lymphoma of the breast associated with a breast implant and seroma (a clinical term used to identify accumulation of fluid around the breast that is derived from serum). We also review the five previously published cases with anaplastic large-cell lymphoma in close proximity to breast implants, to more fully characterize the clinicopathological features of this rare and unusual entity.

\section{Materials and methods}

\section{Patients}

The files of Mayo Clinic Tissue Registry were reviewed for cases of primary lymphoma of the 
breast in association with a breast implant. During the interval 1995-2007, four such patients were identified. Paraffin blocks and/or slides were available on all patients for histological review and for immunohistochemical and molecular genetic studies. All cases were classified according to the current World Health Organization (WHO) classification of malignant lymphomas based on morphology and phenotype. ${ }^{14}$ Primary-care physicians and/ or surgeons who were involved in the care of these patients, were consulted to gather clinical data. The Mayo Clinic Institutional Review Board approved the study.

\section{Immunohistochemistry}

Five-micrometer sections from paraffin-embedded tissue blocks were stained with hematoxylin and eosin (H\&E) and prepared for further immunophenotypic analysis. Immunohistochemistry was performed according to previously described techniques. ${ }^{15}$ Antibodies used are summarized in Table 1.

\section{In Situ Hybridization}

In situ hybridization for the detection of EBV-RNA was performed on formalin-fixed, paraffin-embedded specimens using the procedure described by Chang et al. ${ }^{16}$

\section{Molecular Analysis}

T-cell receptor (TCR) $\gamma$-chain gene rearrangement studies were performed by polymerase chain reaction (PCR) technique, on paraffin-embedded tissue, according to a previously published method. ${ }^{17}$ We used a mixture of four fluorochrome-labeled $5^{\prime}$ primers specific for each of the $T C R \gamma$-chain gene variable region families $(\mathrm{V} \gamma 1, \mathrm{~V} \gamma 2, \mathrm{~V} \gamma 3$ and $\mathrm{V} \gamma 4)$ and two $3^{\prime}$ primers specific for the TCR $\gamma$-chain joining segments (J1 and J2). The PCR products were analyzed on an ABI 3100 using GeneMapper software (Applied Biosystems). TCR PCR was considered positive for a clonal rearrangement if there were three or fewer discrete peaks on the ABI tracing that were at least four times greater in amplitude than the polyclonal background banding pattern.

Immunoglobulin (Ig) gene rearrangement was studied according to a previously published method. ${ }^{18}$

\section{Results}

The clinical features of this patient population are summarized in Table 2 . The mean age of our patient population was 46 years (range, 34-59 years). The mean time between implant placement and lymphoma diagnosis was 5 years (range, 3-7 years), and the mean follow-up time was 13 months (range, 9-20 months).

\section{Clinical Findings}

\section{Patient 1}

A 45-year-old female underwent right mastectomy for an invasive, moderately differentiated, stage IIIB (T4N1M0) ductal carcinoma, and a textured saline implant was placed. Post-surgically, she was treated with chemotherapy and received long-time tamoxifen. A CT scan of her chest 6 years after surgery was unremarkable. However, 1 year later (7 years following surgery) she developed a seroma surrounding the breast implant. The seroma was aspirated and a diagnosis of anaplastic large-cell

Table 1 Immunohistochemistry material

\begin{tabular}{|c|c|c|c|}
\hline Antibody & Clone & Vendor & Location \\
\hline ALK-1 & ALK-1 & Dako & Carpinteria, CA, USA \\
\hline CD2 & AB75 & Novocastra & Burlingame, CA, USA \\
\hline CD3 & Polyclonal & Novocastra & Burlingame, CA, USA \\
\hline CD4 & 4B12 & Novocastra & Burlingame, CA, USA \\
\hline CD5 & $4 \mathrm{C} 7$ & Novocastra & Burlingame, CA, USA \\
\hline CD7 & CD7-272 & Novocastra & Burlingame, CA, USA \\
\hline CD8 & CD8/144B & Dako & Carpinteria, CA, USA \\
\hline CD20 & L26 & Dako & Carpinteria, CA, USA \\
\hline CD30 & Ber-H2 & Dako & Carpinteria, CA, USA \\
\hline CD43 & L60 & BD Biosciences & Palo Alto, CA, USA \\
\hline CD45RB (LCA) & PD7/26 and 2B11 & Dako & Carpinteria, CA, USA \\
\hline TIA-1 & TIA-1 & Immunotech & Fullerton, CA, USA \\
\hline Granzyme B & GRB-7 & Monosan/Caltag & Burlingame, CA, USA \\
\hline$\beta$-F1 & 8A3 & Endogen & Woburn, MA, USA \\
\hline Keratin AE1/AE3 & AE1 and AE3 & Zymed Laboratories Inc. & San Francisco, CA, USA \\
\hline EMA & E29 & Dako & Carpinteria, CA, USA \\
\hline Clusterin & 41D & Upstate Biotechnology & Lake Placid, NY, USA \\
\hline HHV8 & 13B10 & Novocastra & Burlingame, CA, USA \\
\hline PAX5 & 24 & BD Biosciences & Palo Alto, CA, USA \\
\hline
\end{tabular}


Table 2 Clinical features of patients with anaplastic large-cell lymphoma in close proximity to breast implants

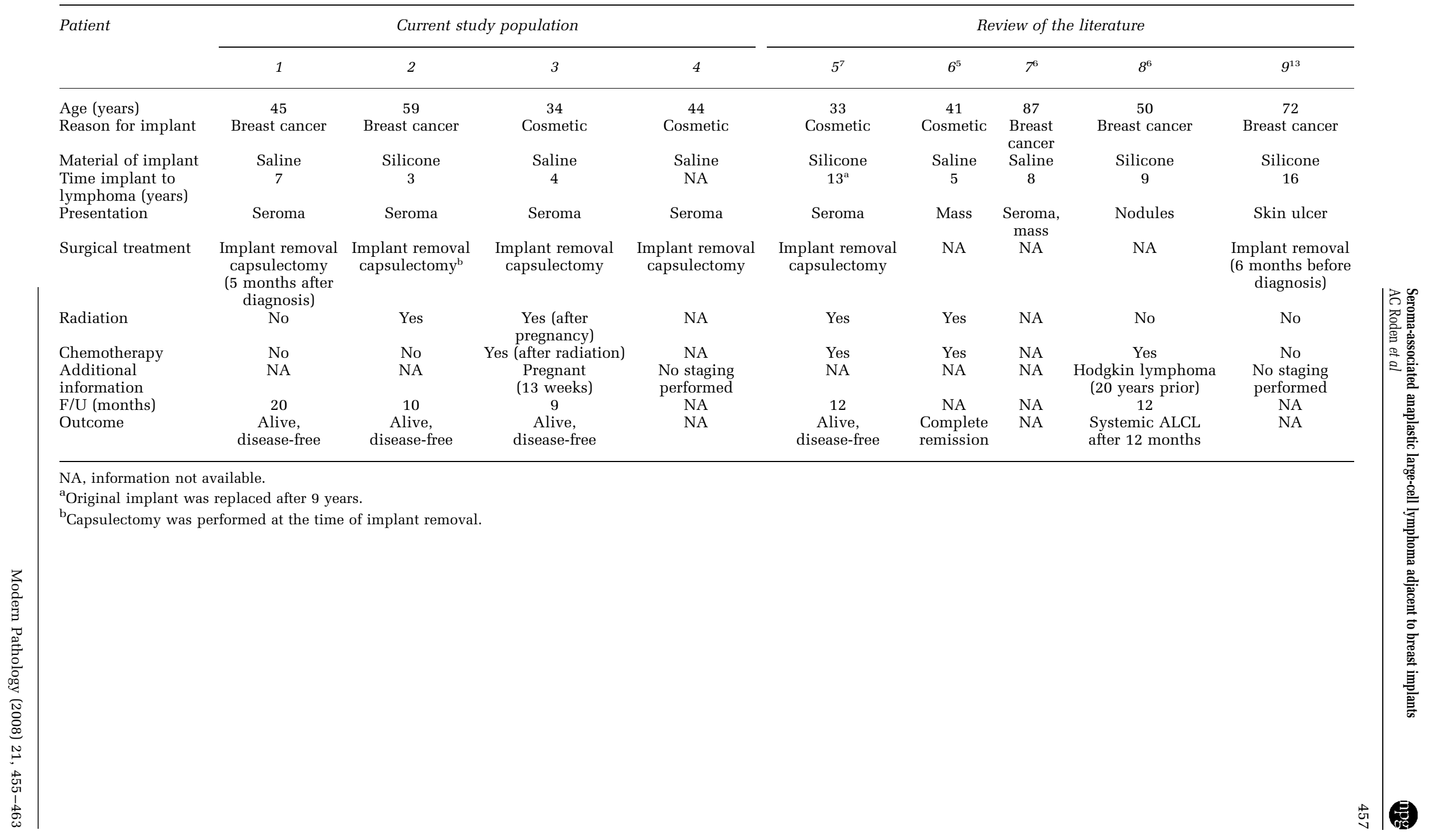


lymphoma was made. The histopathological findings are described below. Immunophenotypic and molecular genetic features are summarized in Table 3.

An MRI of her chest performed at the time of lymphoma diagnosis did not reveal any tumor or lymphadenopathy. The patient declined further treatment other than local application of a homeopathic silver medication. Five months later, faint erythema of the lower reconstructed breast was apparent, which was thought to represent cellulitis/ infection. No lymphadenopathy was apparent. The implant was removed and a partial capsulectomy was performed. To our knowledge, the implant was grossly intact. An outside pathology report described the specimen as having 'no definite evidence of residual lymphoma,' and a culture was negative. The patient was alive and normal 15 months later (20 months after original lymphoma diagnosis), without any signs of recurrence or systemic disease.

\section{Patient 2}

A 59-year-old female underwent mastectomy for breast cancer. Years later, a breast reconstruction with bilateral silicone implants was performed. Three years after breast reconstruction, a seroma developed around the implant on the left side, which was aspirated. Anaplastic large-cell lymphoma was diagnosed. The histopathological findings are described below. Immunophenotypic and molecular genetic features are summarized in Table 3.
One month later, a capsulectomy was performed and the implant was removed. To our knowledge, the implant was grossly intact. Anaplastic large-cell lymphoma was present. The patient subsequently received radiation therapy. The patient was alive and normal 9 months later $(10$ months after lymphoma diagnosis), without any signs of recurrence or systemic disease.

\section{Patient 3}

A 34-year-old female underwent bilateral augmentation mammoplasty with saline implants. Three years later, the left breast appeared larger than the right. At this time it was thought that the right implant might have imploded and was therefore replaced. However, grossly the implant appeared to be intact. One year later, a seroma was diagnosed surrounding the left implant and $700 \mathrm{ml}$ fluid was aspirated three times. Because the patient was about 13 weeks pregnant, both implants were eventually removed out of concerns for the current pregnancy and subsequent lactation. To our knowledge, the implants were grossly intact. The capsulectomy specimen contained anaplastic large-cell lymphoma. The histopathological findings are described below. Immunophenotypic and molecular genetic features are summarized in Table 3.

The patient had no signs of systemic disease. Therapy was not initiated at that time because of her pregnancy. Labor was induced at 33 weeks because of pre-eclampsia. Subsequently, the patient received

Table 3 Immunophenotypic and molecular genetic features of neoplastic cells in anaplastic large-cell lymphomas in close proximity to breast implants

\begin{tabular}{|c|c|c|c|c|c|c|c|c|c|}
\hline \multirow[t]{2}{*}{ Patient } & \multicolumn{4}{|c|}{ Current study population } & \multicolumn{5}{|c|}{ Review of the literature } \\
\hline & 1 & 2 & 3 & 4 & $5^{7}$ & $6^{5}$ & $7^{6}$ & $8^{6}$ & $9^{13}$ \\
\hline Phenotype & $\mathrm{T}$ cell & $\mathrm{T}$ cell & $\mathrm{T}$ cell & $\mathrm{T}$ cell & $\mathrm{T}$ cell & NA & $\mathrm{T}$ cell & T cell & $\mathrm{T}$ cell \\
\hline ALK-1 & - & - & - & - & - & NA & - & - & - \\
\hline CD2 & Focal+ & + & Focal+ & - & + & NA & NA & + & NA \\
\hline CD3 & Weak+ & + & Weak+ & + & - & NA & Focal+ & Weak+ & - \\
\hline CD4 & + & Focal+ & Weak+ & + & NA & NA & + & NA & Weak+ \\
\hline CD5 & + & - & - & - & NA & NA & - & Weak+ & Weakt \\
\hline CD7 & Focal+ & NA & NA & Focal+ & NA & NA & NA & NA & NA \\
\hline CD8 & Focal+ & + & - & - & NA & NA & - & NA & - \\
\hline CD20 & NA & - & - & - & - & NA & NA & - & - \\
\hline CD30 & + & + & + & + & + & + & + & + & + \\
\hline CD43 & + & + & NA & + & + & NA & + & Weak+ & - \\
\hline CD45 & + & + & + & + & NA & NA & + & + & - \\
\hline TIA-1 & + & + & + & + & NA & NA & + & NA & NA \\
\hline Granzyme B & + & + & + & + & NA & NA & NA & NA & NA \\
\hline EMA & + & + & + & + & Focal+ & NA & Focal+ & - & - \\
\hline Clusterin & Subset+ & Subset+ & Subset+ & Subset+ & NA & NA & NA & NA & NA \\
\hline PAX 5 & - & - & - & - & NA & NA & NA & NA & - \\
\hline$\beta$-F1 & - & - & - & - & NA & NA & NA & NA & NA \\
\hline Keratin & NA & - & - & - & NA & NA & - & NA & - \\
\hline TCR $\gamma$-chain rearrang & Clonal & Polyclonal & Clonal & Clonal & Clonal & NA & Clonal & NA & NA \\
\hline IgH rearrang & Polyclonal & Polyclonal & NA & NA & Polyclonal & NA & NA & NA & NA \\
\hline EBV & - & - & - & - & - & NA & NA & NA & - \\
\hline HHV8 & - & - & - & - & - & NA & NA & NA & NA \\
\hline
\end{tabular}

'+', expressed; '-', not expressed; NA, information not available; rearrang, rearrangement. 
six cycles of CHOP chemotherapy followed by radiation treatment. She was alive and well 9 months after her lymphoma diagnosis, without any signs of recurrence or systemic disease.

\section{Patient 4}

A 44-year-old female underwent augmentation mammoplasty with a saline implant. The time of implant is not known to us. Eventually a seroma was noticed around the left implant. Fluid was aspirated and a diagnosis of anaplastic large-cell lymphoma was made. The histopathological findings are described below. Immunophenotypic and molecular genetic findings are summarized in Table 3. Three months later, capsulectomy was performed and the implant was removed. By report, the implant was grossly intact. The capsulectomy specimen showed fibrosis with nonspecific chronic inflammation and foreign body giant-cell reaction. There was no evidence of lymphoma.

Because this diagnosis was made recently, staging has not yet been performed and no treatment decisions have been made.

\section{Histopathological, immunophenotypic and molecular genetic findings}

The histopathological findings were similar in all four patients. In all patients the pathological specimen examined was the 'seroma' adjacent to the breast implant. Histological and cytological preparations showed a population of non-cohesive large cells with pleomorphic nuclei, dispersed chromatin, occasionally prominent nucleoli and variably abundant eosinophilic cytoplasm exhibiting indistinct cell borders suspended in a serous/fibrinous background (Figures 1a and b). There were frequent mitoses. No infiltration of surrounding solid tissues was evident in the specimens available for pathological examination. There was no evidence of refractile material that could have indicated leaking silicone.

The immunophenotype of the neoplastic cells and molecular genetic findings for each individual patient are summarized in Table 3. In all patients, neoplastic cells demonstrated a T-cell phenotype expressing CD3 (Figure 1c), CD4 and CD45 (Figure 1d) at variable intensity. In some patients, CD2 $(n=1)$ or CD5 expression $(n=2)$ was aberrantly lost and there was aberrant coexpression of CD8 in two patients. All tumors were strongly positive for CD30 (Figure 1e), TIA-1 (Figure 1f), granzyme B (Figure 1g) and EMA. A subset of neoplastic cells in each specimen showed Golgi-staining pattern for clusterin. However, all specimens lacked staining for anaplastic lymphoma kinase-1 (ALK-1) (Figure 1h), $\beta$-F1 and HHV8. In situ hybridization was negative for EBV.

Clonal TCR $\gamma$-chain gene rearrangements were detected in three patients (Figure 2); the fourth patient had a polyclonal pattern. Ig gene rearrangement studies had a polyclonal pattern in the two patients tested.

\section{Literature review}

Primary anaplastic large-cell lymphoma of the breast in association with breast implant has been reported in five female patients. ${ }^{5-7,13}$ The available clinical data for these patients are summarized in Table 2 . The mean age of the patients was 57 years (range, 33-87 years). The mean time elapsed between placement of the breast implant and diagnosis of lymphoma was 10 years (range, 5-16 years). No systemic disease was identified at the time of diagnosis in two patients, whereas no staging was provided for the other three. Three patients were treated with chemotherapy, two of whom also received radiation therapy. One patient declined any further treatment and there was no information on treatment provided for the remaining patients. Follow-up data were provided, in part, for three patients. One patient who was treated with chemotherapy and radiation therapy, was alive after 12 months without any evidence of disease. One patient achieved complete remission after chemotherapy and radiation therapy, but the time of follow-up was not provided. One patient treated with chemotherapy developed pleural and pericardial effusions, lung infiltrates and mediastinal lymphadenopathy 12 months after her diagnosis of anaplastic large-cell lymphoma. Analysis of the pleural fluid confirmed relapsed anaplastic largecell lymphoma. Of further interest, this last patient had a history of Hodgkin lymphoma 20 years before diagnosis of anaplastic large-cell lymphoma.

The available immunophenotypic and molecular genetic data for the five patients are summarized in Table 3. The tumor cells were positive for CD30 in all patients, and an ALK-1-negative T-cell phenotype was detected in all patients tested $(n=4)$. Clonal TCR $\gamma$-chain gene rearrangements were detected in both patients for whom this testing was performed.

\section{Discussion}

Although the majority of lymphomas in the breast have a B-cell phenotype, most breast implantassociated lymphomas have a T-cell phenotype. Anaplastic large-cell lymphoma is the most common T-cell lymphoma in close proximity to a breast implant, as nine of $13(69 \%)$ of these in this study and in the literature belong to this category. ${ }^{5-7,13}$

Two types of anaplastic large-cell lymphoma are defined in the WHO classification of malignant lymphomas: (1) systemic anaplastic large-cell lymphoma and (2) primary cutaneous anaplastic largecell lymphoma. ${ }^{14}$ In $60-85 \%$ of systemic anaplastic large-cell lymphomas, the pathogenesis is linked to phosphorylation of a tyrosine kinase, the ALK-1. ALK-1 protein expression is most commonly seen as 

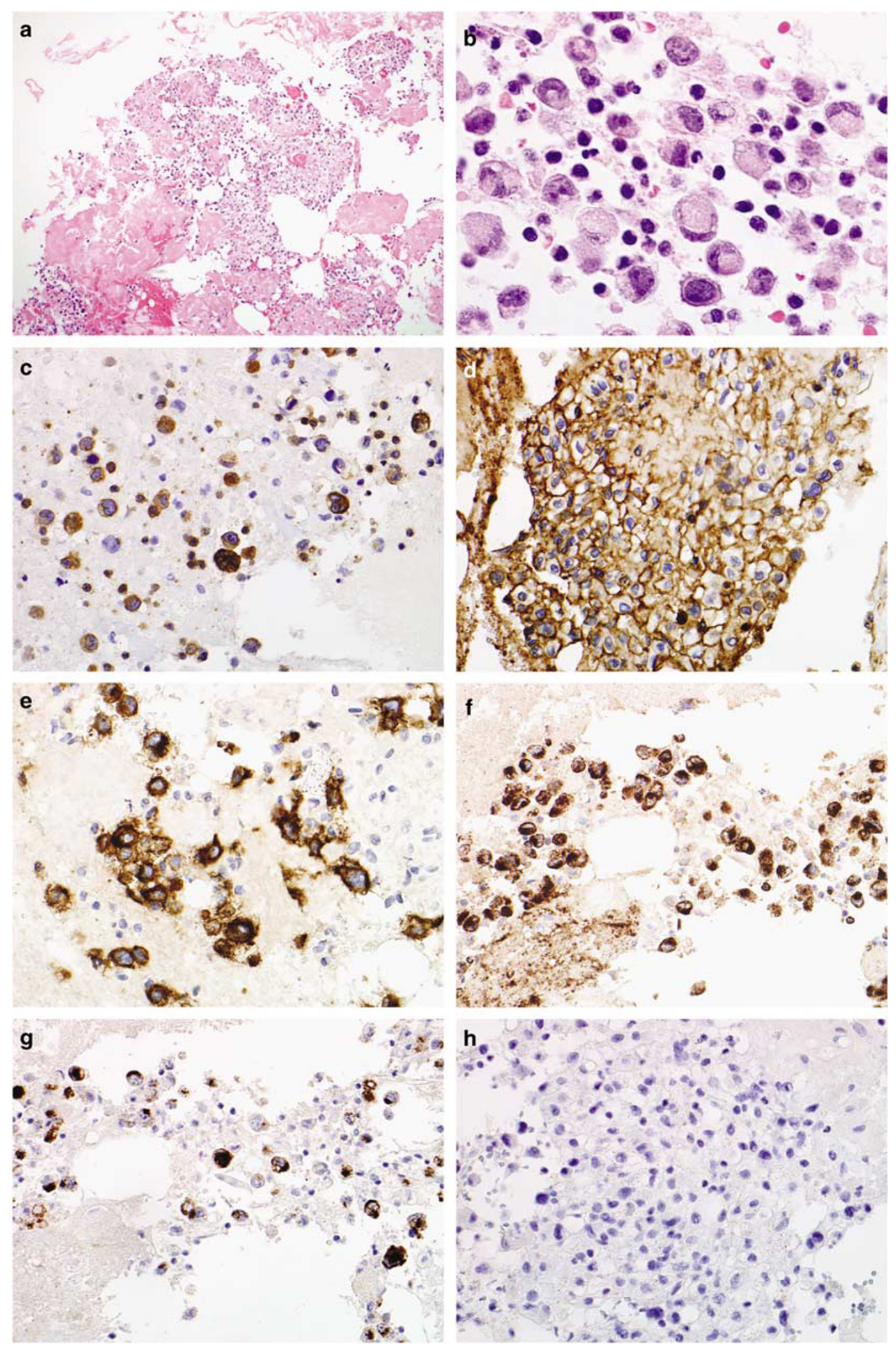

Figure 1 Representative capsulectomy specimen from anaplastic large-cell lymphoma adjacent to breast implant (case 4). An infiltrate of atypical cells suspended in serous/fibrinous material (H\&E) (a). The neoplastic cells are large and have pleomorphic cytological features (H\&E) (b). Neoplastic cells stain for CD3 (c) and uniformly express CD45 (d). They are strongly positive for CD30 (e), TIA-1 (f) and granzyme B (g). Neoplastic cells lack ALK-1 expression (h). Original magnifications: $\times 100(\mathbf{a}) ; \times 1000-\mathrm{oil}(\mathbf{b}) ; \times 400(\mathbf{c}-\mathbf{h})$. 


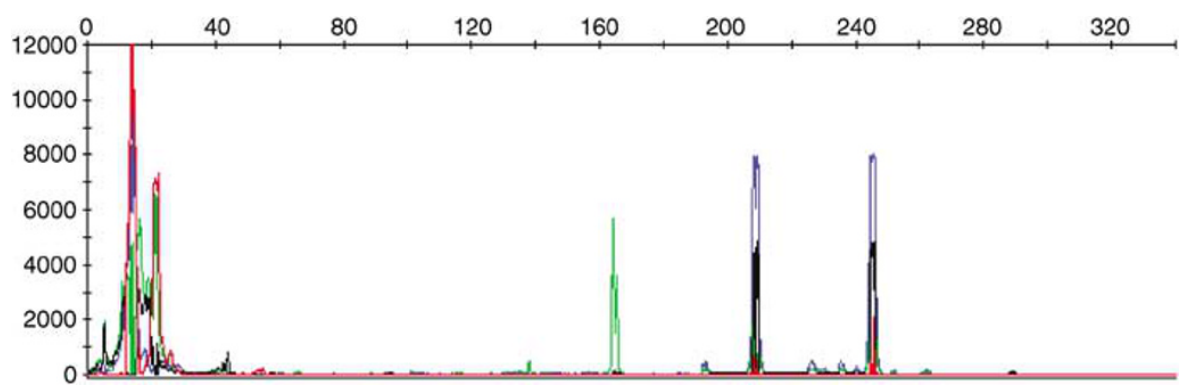

Figure 2 Representative tracings from TCR $\gamma$-chain gene rearrangement studies performed by PCR technique on specimen from patient 3 showing positive peaks, indicating clonality in the neoplastic cells. Colors are characteristic for certain primer sets.

a result of $\mathrm{t}(2 ; 5)(\mathrm{p} 23 ; \mathrm{q} 35)$, which fuses the $A L K$ gene on 2 p23 to the nucleophosmin $(N P M)$ gene on $5 q 35$, resulting in constitutive activation of the ALK kinase domain. ${ }^{19}$ ALK-1-positive systemic anaplastic large-cell lymphomas occur most often in individuals less than 30 years of age, with a peak incidence in childhood. ALK-1-negative systemic anaplastic large-cell lymphomas tend to occur in an older group of patients than those with ALK-1positive anaplastic large-cell lymphomas. Polychemotherapy is the current treatment approach for both ALK-1-positive and ALK-1-negative systemic anaplastic large-cell lymphomas. However, overall and event-free survival are significantly better for ALK-1-positive than for ALK-1-negative systemic anaplastic large-cell lymphomas, with 5year survival rates ranging between 71 and 100\% and 15 and $45 \%$, respectively. ${ }^{19}$

Primary cutaneous anaplastic large-cell lymphomas arise de novo in the skin and usually affect elderly patients who present with ulceration and erythema of the skin. Primary cutaneous anaplastic large-cell lymphomas appear to have a different pathogenesis than systemic anaplastic large-cell lymphomas. ${ }^{20}$ ALK-1 expression is extremely rare in these tumors. ${ }^{19}$ Patients with the primary cutaneous form have no evidence of systemic disease for at least 6 months after presentation, and spontaneous regression occurs in approximately $25 \%$ of patients. Excision or local irradiation is usually effective treatment. Five-year survival for primary cutaneous anaplastic large-cell lymphoma is greater than $90 \% .^{20}$

Nine patients with breast implant-associated anaplastic large-cell lymphomas typically presented with a seroma around the implant or with a mass and had stage $1 \mathrm{E}$ disease. Although the follow-up time for these patients was somewhat short, the lack of recurrence or development of systemic disease in all but one patient is noteworthy. Based on the combination of anaplastic cytology, T-cell phenotypic aberrancy, including expression of clusterin, and clonal TCR $\gamma$-chain gene rearrangements, we concluded that these cases are malignant rather than reactive despite the 'benign' clinical course. Interestingly, the single patient with eventual systemic dissemination presented with multiple nodules rather than seroma, indicating a distinct pathogenesis. In all patients, the neoplastic cells had been uniformly negative for ALK-1 expression. These observations suggest that anaplastic large-cell lymphomas adjacent to breast implants have a pathogenesis more analogous to primary cutaneous anaplastic large-cell lymphoma rather than systemic ALK-negative anaplastic large-cell lymphoma. Interestingly, cutaneous erythema overlying the implant or skin ulceration above the implant was observed in two patients, which emphasizes the proximity of these implant-associated lymphoproliferative lesions to the skin.

Morphologically, there might be overlap of anaplastic large-cell lymphoma with Hodgkin lymphoma because cases of Hodgkin lymphoma, particularly nodular sclerosis type, may contain confluent sheets of CD30-positive Reed Sternberg cell variants resembling anaplastic large-cell lymphoma. However, the T-cell phenotype of neoplastic cells that coexpress EMA and cytotoxic granuleassociated proteins, the lack of PAX5 expression and the negativity for EBV in all of our specimens support the diagnosis of anaplastic large-cell lymphoma. Furthermore, a subset of neoplastic cells in all our specimens showed the Golgi-staining pattern for clusterin that is commonly described in primary cutaneous anaplastic large-cell lymphomas. ${ }^{21-23}$ This finding further supports that the pathogenesis of implant-associated breast anaplastic large-cell lymphomas resembles that of primary cutaneous anaplastic large-cell lymphoma. Because implantassociated breast lymphomas commonly present as therapy-resistant seroma, primary effusion lymphoma might also be considered in the differential diagnosis. However, we show that neoplastic cells lack staining for HHV8 and EBV, basically excluding the diagnosis of primary effusion lymphoma.

Silicone was introduced 30 years ago when it was believed to be biologically inert and to lack tissue immunogenicity. ${ }^{24}$ However, some case reports have attributed various immunological side effects to the use of silicone, such as localized and distant granulomatous inflammation in the breast, reactive lymphadenopathy, inflammation of joint capsules following erosion or fracture of a prosthesis, endocarditis, nephropathy, hepatic granulomas and 
fibrosis, respiratory distress syndrome and human adjuvant disease, indicating that silicone is not inert and can, in fact, provoke a local or systemic immune response. ${ }^{25-30}$ Furthermore, silicone gel-protein mixtures have been used as potent immunological adjuvants. ${ }^{31}$ In support of this, evidence from animal studies suggests that silicone gel is immunogenic ${ }^{32}$ and can induce plasmacytomas in genetically predisposed mice. ${ }^{33}$ Additionally, Cook et al. ${ }^{11}$ reported a case of follicular lymphoma, adjacent to a foreignbody granulomatous response to a silicone breast implant. It has been shown that silicone gels contain several components that are potentially biologically active, such as residual vinyl groups and platinum. However, silicone gel liquids can leak out of the capsules either by rupture or seepage. Even in the absence of detectable rupture, it has been shown that leakage is common, as evidenced by the finding of intracellular (macrophages) and extracellular silicone in the superficial portion of the fibrovascular tissue reaction that comprises the periprosthetic breast capsule. Saline implants are also often surrounded by an impermeable silicone elastomeric capsule that by itself might be immunogenic. Among the nine patients with breast implant-associated anaplastic large-cell lymphoma, five had saline and four had silicone implants. However, whether the saline implants had a silicone elastomeric capsule or a polyurethane-walled capsule is not known to us. Although grossly there was no rupture apparent in our patients or in any of the patients reported in the literature, leakage of silicone from the implant or the implant capsule might have occurred. Overall, we did not find an apparent difference in overall clinical presentation, morphology and immunophenotype of the neoplastic cells, or of clinical outcome between patients with silicone vs saline implants.

Anaplastic large-cell lymphomas are lymphomas of activated lymphocytes. ${ }^{20}$ In light of this, the development of anaplastic large-cell lymphoma in the vicinity of breast implants might be a consequence of an immunological reaction to the silicone. For instance, $\mathrm{T}$ cells might be stimulated by the silicone and eventually become clonally expanded by a mechanism that has not yet been defined. Once the implant and the tumor are removed, the $\mathrm{T}$ cells may lack the immunological stimulus to expand further.

In summary, it is important to be aware that lymphomas can occur in the vicinity of breast implants independent of the implant-material used. Therapy-resistant seroma in the proximity of or surrounding a breast implant should raise the level of suspicion for the presence of a lymphoma. ALK-1negative T-cell anaplastic large-cell lymphomas are the most common breast implant-associated lymphomas. The clinicopathological features of these tumors cited herein suggest that they are more like primary cutaneous anaplastic large-cell lymphomas than ALK-1-negative systemic anaplastic large-cell lymphomas.

\section{References}

1 Cohen PL, Brooks JJ. Lymphomas of the breast. A clinicopathologic and immunohistochemical study of primary and secondary cases. Cancer Immunol Immunother 1991;67:1359-1369.

2 Vasei M, Kumar PV, Malekhosseini SA, et al. Primary T-cell lymphoma of the breast with lymphoepithelial lesion. A case report. APMIS 1997;105:445-448.

3 Lin Y, Govindan R, Hess JL. Malignant hematopoietic breast tumors. Am J Clin Pathol 1997;107:177-186.

4 Aguilera NS, Tavassoli FA, Chu WS, et al. T-cell lymphoma presenting in the breast: a histologic, immunophenotypic and molecular genetic study of four cases. Mod Pathol 2000;13:599-605.

5 Keech Jr JA, Creech BJ. Anaplastic T-cell lymphoma in proximity to a saline-filled breast implant. Plast Reconstr Surg 1997;100:554-555.

6 Gaudet G, Friedberg JW, Weng A, et al. Breast lymphoma associated with breast implants: two case-reports and a review of the literature. Leuk Lymphoma 2002;43:115-119.

7 Sahoo S, Rosen PP, Feddersen RM, et al. Anaplastic large cell lymphoma arising in a silicone breast implant capsule: a case report and review of the literature. Arch Pathol Lab Med 2003;127:e115-e118.

8 Duvic M, Moore D, Menter A, et al. Cutaneous T-cell lymphoma in association with silicone breast implants. J Am Acad Dermatol 1995;32:939-942.

9 Sendagorta E, Ledo A. Sezary syndrome in association with silicone breast implant. J Am Acad Dermatol 1995;33:1060-1061.

10 Said JW, Tasaka T, Takeuchi S, et al. Primary effusion lymphoma in women: report of two cases of Kaposi's sarcoma herpes virus-associated effusion-based lymphoma in human immunodeficiency virus-negative women. Blood 1996;88:3124-3128.

11 Cook PD, Osborne BM, Connor RL, et al. Follicular lymphoma adjacent to foreign body granulomatous inflammation and fibrosis surrounding silicone breast prosthesis. Am J Surg Pathol 1995;19:712-717.

12 Kraemer DM, Tony HP, Gattenlohner S, et al. Lymphoplasmacytic lymphoma in a patient with leaking silicone implant. Haematologica 2004;89:ELT01.

13 Fritzsche FR, Pahl S, Petersen I, et al. Anaplastic largecell non-Hodgkin's lymphoma of the breast in periprosthetic localisation 32 years after treatment for primary breast cancer-a case report. Virchows Arch 2006;449:561-564.

14 Jaffe ES, Harris NL, Stein H, et al. Pathology and Genetics of Tumours of Haematopoietic and Lymphoid Tissues. IARC Press: Lyon, 2001.

15 Kurtin P, Hobday K, Ziesmer S. Demonstration of distinct antigenic profiles of small B-cell lymphomas by paraffin section immunohistochemistry. Am J Clin Pathol 1999;112:319-329.

16 Chang KL, Chen YY, Shibata D, et al. Description of an in situ hybridization methodology for detection of Epstein-Barr virus RNA in paraffin-embedded tissues, with a survey of normal and neoplastic tissues. Diagn Mol Pathol 1992;1:246-255. 
17 Morice WG, Katzmann JA, Pittelkow MR, et al. A comparison of morphologic features, flow cytometry, TCR-Vbeta analysis, and TCR-PCR in qualitative and quantitative assessment of peripheral blood involvement by Sezary syndrome. Am J Clin Pathol 2006; 125:364-374.

18 McClure RF, Kaur P, Pagel E, et al. Validation of immunoglobulin gene rearrangement detection by PCR using commercially available BIOMED-2 primers. Leukemia 2006;20:176-179.

19 ten Berge RL, Oudejans JJ, Ossenkoppele GJ, et al. ALK expression in extranodal anaplastic large cell lymphoma favours systemic disease with (primary) nodal involvement and a good prognosis and occurs before dissemination. J Clin Pathol 2000;53:445-450.

20 Kadin ME, Carpenter C. Systemic and primary cutaneous anaplastic large cell lymphomas. Semin Hematol 2003;40:244-256.

21 Lae ME, Ahmed I, Macon WR. Clusterin is widely expressed in systemic anaplastic large cell lymphoma but fails to differentiate primary from secondary cutaneous anaplastic large cell lymphoma. Am J Clin Pathol 2002;118:773-779.

22 Kumar S, Pittaluga S, Raffeld M, et al. Primary cutaneous CD30-positive anaplastic large cell lymphoma in childhood: report of 4 cases and review of the literature. Pediatr Dev Pathol 2005;8:52-60.

23 Nascimento AFPJ, Pinkus GS. Clusterin, a marker for anaplastic large cell lymphoma immunohistochemical profile in hematopoietic and nonhematopoietic malignant neoplasms. Am J Clin Pathol 2004;121:709-717.
24 Nosanchuk J. Injected dimethylpolysiloxane fluid: a study of antibody and histologic response. Plast Reconstr Surg 1968;42:562-566.

25 Baldwin Jr CM, Kaplan EN. Silicone-induced human adjuvant disease? Ann Plast Surg 1983;10:270-273.

26 Gordon M, Bullough PG. Synovial and osseous inflammation in failed silicone-rubber prostheses. J Bone Joint Surg Am 1982;64:574-580.

27 Kumagai Y, Abe C, Shiokawa Y. Scleroderma after cosmetic surgery: four cases of human adjuvant disease. Arthritis Rheum 1979;22:532-537.

28 Travis WD, Balogh K, Wolf BC, et al. Silicone-induced endocarditis. A complication of transvenous cardiac pacing catheterization. Arch Pathol Lab Med 1986; 110:51-54.

29 Travis WD, Balogh K, Abraham JL. Silicone granulomas: report of three cases and review of the literature. Hum Pathol 1985;16:19-27.

30 Dodd LG, Sneige N, Reece GP, et al. Fineneedle aspiration cytology of silicone granulomas in the augmented breast. Diagn Cytopathol 1993;9: 498-502.

31 Naim JO, Ippolito KM, van Oss CJ. Adjuvancy effect of different types of silicone gel. J Biomed Mater Res 1997;37:534-538.

32 Yoshida SH, Swan S, Teuber SS, et al. Silicone breast implants: immunotoxic and epidemiologic issues. Life Sci 1995;56:1299-1310.

33 Potter M, Morrison S, Miller F. Induction of plasmacytomas in genetically susceptible mice with silicone gels. Curr Top Microbiol Immunol 1995;194:83-91. 